COMMENT. Long-term treatment with steroids in DMD patients results in an increase in duration of independent ambulation of more than 3 years and a significantly decreased risk of scoliosis. Adverse effects include a vertebral compression fracture in onethird of steroid-treated patients, although $80 \%$ are asymptomatic and are discovered incidentally at scoliosis screening. The risk of long bone fracture is 2.6 times greater in treated compared to untreated patients. The increased fracture rate is linked to prolonged independent ambulation and increased body weight. All treated patients are prescribed calcium supplements and have bone density measurements.

\title{
DOK7 MUTATIONS IN CONGENITAL MYASTHENIC SYNDROMES
}

Clinical and genetic data of 14 patients from 12 congenital myasthenic syndrome (CMS) kinships with mutations in the DOK ('downstream-of-kinase') 7 gene are presented by researchers from the Friedrich-Baur-Institute, Munich, Germany, and several other centers. The chinical presentation of CMS with DOK7 mutations was variable. Typically, onset of symptoms was in the $2^{\text {nd }}$ year of life, with an awkward or waddling gait. Four patients were hypotonic at birth, whereas 3 developed symptoms in the $2^{\text {nd }}$ or $3^{\text {rd }}$ decade. The majority (12 of 14) had bilateral ptosis but no paresis of eye movements. Facial and bulbar weakness occurred in $9(64 \%)$, and several had difficulties in chewing and swallowing. Respiratory function was impaired in $10(71 \%)$, and 2 with onset at birth needed assisted ventilation in the first months of life. Most developed scoliosis, and some had general or selective muscle atrophy. All but 1 had moderate to severe proximal muscle weakness, and a waddling 'sinuous gait' (inward rotation of the knees). Weakness was exercise-dependent, and fluctuated over long periods. In some, symptoms were progressive, with loss of ambulation and respiratory insufficiency in adolescence, whereas in others, severity of symptoms remained constant. AChR antibody testing was negative, and creatine kinase levels were normal. Muscle biopsies showed only nonspecific mild myopathic changes, and no tubular aggregates. Intravenous Edrophonium test was positive in 5 (35.5\%), but none benefited from long-term therapy with esterase inhibitors, and some even worsened. Patients with CMS and mutations in other genes differed from CMS with $D O K 7$ mutations. With CHRNE mutations, eye movements are frequently affected, and with CHAT and RAPSN mutations, episodic apneas occur at birth. In contrast, with $D O K 7$ mutations eye movements are usually spared except for ptosis, and respiratory function shows a progressive deterioration. Limb-girdle myasthenia, unlike DOK7 patients is benefited by esterase inhibitor therapy. (Muller JS, Herczegfalvi A, Vilchez JJ et al. Phenotypical spectrum of DOK7 mutations in congenital myasthenic syndromes. Brain 2007;130:1497-1506). (Respond: Hanns Lochmuller MD, Friedrich-Baur-Institute, Molecular Myology Lab, Marchioninistrasse 17, 81377 Munchen, Germany).

COMMENT. The DOK7 mutation congenital myasthenic syndrome has a broad clinical phenotype, with onset varying from birth to adult life. Features distinguishing the $D O K 7$ mutation patients from other known mutation phenotypes include the absence of external ophthalmoplegia, a progressive deterioration of respiratory function, and lack of long-term response to esterase inhibitors.

Since the description of a congenital myasthenic syndrome in 6 infants treated at the Massachusetts General Hospital in 1960, a variety of molecular causes for the syndrome have 
been identified, including endplate acetylcholine and AChR deficiencies, a slow-channel syndrome, and defects in resynthesis of ACh and kinetics of AChR.

The common feature of CMS is a defect in the neuromuscular junction with exerciseinduced weakness of skeletal muscle. In the initial report, cases presented with ptosis, weak cry, and generalized weakness. Diagnosis was delayed until 1 to 4 years after birth, when ptosis and external ophthalmoplegia were the most prominent signs, and generalized weakness and respiratory difficulty were mild in degree. Response to cholinergic drug therapy was poor, and ophthalmoplegia was unrelieved. Except for the resistance to treatment, CMS with $D O K 7$ mutations has an unusual clinical phenotype.

\section{SLEEP DISORDERS}

\section{DYSSOMNIAS AND PARASOMNIAS}

The prevalence of dyssomnias and parasomnias was determined in a longitudinal study of a sample of 2223 infants born in 1997-98 in the province of Quebec, Canada, and reported from the Sleep Disorders Center, Sacre-Coeur Hospital, University of Montreal. Self-administered questionnaires were completed by the mothers when the children reached 2.5 years of age, and at intervals up to 6 years. Dyssomnias manifested by frequent night wakings $(-/>1$ per night) and difficulty falling asleep at night $(-/>30 \mathrm{~min})$ were reported in $36.3 \%$ and $12.2 \%$ children at age 2.5 years, respectively. At age 6 years, the percentage with frequent night wakings had decreased steadily to $13.2 \%$, and the percentage with difficulty falling asleep had decreased significantly to $7.4 \%$. Between 2.5 and 6 years, the percentage with difficulty falling asleep had first increased to $16.0 \%$ at ages 3.5 and 4 and then decreased to $10 \%$ at age 5 and to $7.4 \%$ at age 6 .

The overall prevalence of parasomnias from 2.5 to 6 years was as follows: somnambulism, $14.5 \%$; sleep terrors, $39.8 \%$; somniloquy (talking during sleep), $84.4 \%$; enuresis, $25.0 \%$; bruxism, $45.6 \%$; and rhythmic movements, $9.2 \%$. Sleep terrors were more frequent at ages $2.5,3.5$, and 4 than at ages 5 and 6 . Rhythmic movements (body-rocking and head-banging) were more prevalent at age 2.5 than at all later ages. Boys outnumbered girls (ratio 2:1) with somnambulism and enuresis, but no gender differences were observed for prevalence of other parasomnias. Persistent somnambulism was correlated with persistent sleep terrors $(\mathrm{P}<.01)$ and persistent somniloquy $(\mathrm{P}<.001)$. Persistent sleep terrors were correlated with persistent somniloquy $(\mathrm{P}<.001)$, and with frequent night wakings $(\mathrm{P}<.001)$.

Children with persistent dyssomnias had higher separation anxiety scores, and had been put to bed already asleep in a greater proportion.at 2.5 years. Sleep-onset difficulties were associated with low socioeconomic status (SES), but not with co-sleeping or separation anxiety. Neither dyssomnia was associated with ADHD. Persistent parasomnias were associated with separation anxiety. They had little impact on sleep duration. Persistent somnambulism was related to a high ADHD score. Sleep terrors were related to a recent divorce of parents. Persistent rhythmic movements were related to insufficient SES and maternal depression. (Petit D, Touchette E, Trembley RE et al. Dysomnias and Parasomnias in early childhood. Pediatrics May 2007;119:e1016-e1025). (Respond: Jacques Montplaisir MD, PhD, Sleep Disorders Center, Sacre-Coeur Hospital, 5400 Gouin Blvd W, Montreal, Quebec, Canada H4J 1CS). 